\title{
Topological Equivalence of a Structure-Preserving Power Network Model and a Non-Uniform Kuramoto Model of Coupled Oscillators
}

\author{
Florian Dörfler and Francesco Bullo
}

\begin{abstract}
We study synchronization in the classic structurepreserving power network model proposed by Bergen and Hill. We find that, locally near the synchronization manifold, the phase and frequency dynamics of the power network model are topologically conjugate to the phase dynamics of a non-uniform Kuramoto model and decoupled exponentially stable dynamics for the frequencies. This topological conjugacy implies the equivalence of local exponential synchronization in power networks and in non-uniform Kuramoto oscillators. Hence, we can harness the results available for Kuramoto oscillators to analyze synchronization in power networks. We establish necessary and sufficient conditions for phase synchronization, sufficient conditions for frequency synchronization, and necessary and sufficient conditions for frequency synchronization with a uniform topology. These conditions also extend the results known for the classic first-order Kuramoto model and the second-order linear consensus protocols. Our synchronization conditions all share a common physical interpretation: the ratio of power inputs and dissipation has to be sufficiently uniform and the coupling in the network has to be sufficiently strong.
\end{abstract}

\section{INTRODUCTION}

The vast North American interconnected power grid is often referred to as the largest and most complex machine engineered by humankind. Local instabilities arising in such a complex system can trigger cascading failures and ultimately result in wide-spread blackouts. In face of the rising complexity of the envisioned future power grid and the stochastic disturbances caused by renewables such as wind and solar power, the understanding of the system complexity and stability becomes more and more important.

Power system stability is broadly subdivided into rotor angle and voltage stability. Rotor angle stability is the ability of the power system to remain in synchronism when subjected to disturbances, and it is further classied as transient stability for severe disturbances. Generally, the complexity of a power network and the related (in)stability issues are not understood [1]. In particular, an open problem recognized by the power system community and not resolved yet by classical methods is the quest for explicit and concise conditions relating transient stability to the parameters and graph-theoretical properties of the underlying network [2]. In [3] we provided a solution to this problem for a network-reduced power system model with non-zero transfer conductances by means of a singular perturbation analysis and by using tools from coupled oscillators and consensus networks. These results

This work was supported in part by NSF grants IIS-0904501 and CNS0834446 .

Florian Dörfler and Francesco Bullo are with the Center for Control, Dynamical Systems and Computation, University of California at Santa Barbara, Santa Barbara, CA 93106, \{dorfler, bullo\}eengineering.ucsb.edu on the reduced network can then be related to the original network via Kron reduction and graph theory [4].

In 1981 Bergen and Hill proposed a structure-preserving power network model [5] to represent the network components and topology explicitly and to overcome the difficulties in the analysis of network-reduced models with non-neglibile transfer conductances. Since the structure-preserving power network model can be cast as mixed Hamiltonian and gradient-like system, the traditional transient stability analysis methods are based on Hamiltonian arguments together with computational tools [6]. Even though these Hamiltonian methods are very powerful, in particular to estimate the region of attraction of synchronous equilibria, they do not provide concise conditions for synchronization.

Synchronization recently attracted lots of interest in the control, dynamical systems, and physics communities. Especially, the simple and yet rich dynamics of the coupled oscillator model proposed by Kuramoto [7] have often been studied as a prototypical example for synchronization. The Kuramoto model finds application in various biological and technological synchronization phenomena, and we refer the reader to [8], [9], [10] for various applications, further references, and theoretic results. As observed in [3] and references therein, a power network can be cast as a second-order Kuramoto model with inertia, viscous frequency damping, non-complete coupling topology, and non-identical natural frequencies. Such a Kuramoto model has been studied computationally in [9] and references therein, where the inertial effects have been found to inhibit synchronization. The second-order consensus protocols studied in [11] and references therein can also be seen as a linearized version of the power network model with identical nodal dynamics. For both the Kuramoto model and consensus protocol the relation between synchronization and the parameters and topology of the underlying network is very well understood.

The contributions of this paper are three-fold.

First, we show that, locally near the synchronization manifold, the phase and frequency dynamics of the power network model are topologically conjugate to the phase dynamics of a non-uniform Kuramoto model and decoupled exponentially stable dynamics for the frequencies. The two decoupled dynamics correspond exactly to the fast and slow dynamics found via singular perturbation analysis in [3]. Compared to [3], this local topological conjugacy result holds without any assumptions on the system parameters such as a sufficiently strong damping. Moreover, the following three statements are found to be equivalent: exponential synchronization in the structure-preserving power network model, exponential synchronization in the non-uniform Kuramoto 
model, and exponential stability of a topological Kuramoto model. Hence, we can combine the tools provided by the power networks community and the literature on coupled Kuramoto oscillators to prove local synchronization.

Second, we address the outstanding problem proposed in [2] and provide novel and explicit conditions relating synchronization in the structure-preserving power model to the underlying network parameters and topology. Our conditions are necessary and sufficient for phase synchronization and sufficient for frequency synchronization. For a complete and uniform coupling graph, our conditions are also necessary and sufficient for frequency synchronization. In each case, the convergence rate of synchronizing solutions is exponential, we can give bounds on the ultimate phase cohesiveness, and our synchronization conditions can be interpreted as "the ratio of power inputs and dissipation has to be sufficiently uniform and the coupling in the network has to be sufficiently strong", which confirms our results on the reduced model [3].

Third and finally, our exact synchronization conditions for a power network with a complete and uniform coupling graph also extend the results for the classic Kuramoto model and the consensus protocol. In particular, our synchronization conditions are exact for multi-rate Kuramoto models and second-order Kuramoto models with inertia and viscous frequency damping. Contrary to the numeric observations listed in [9], we prove that the inertia do not affect the necessary and sufficient local synchronization conditions. Furthermore, our approach extends the results [11], where the eigenvalues of the second-order consensus protocol are related to those of the corresponding first-order model. We provide a natural generalization of this result to the nonlinear regime: the second-order Kuramoto model achieves phase (resp. frequency) synchronization if and only if the corresponding first-order non-uniform Kuramoto model synchronizes.

This article is organized as follows. The remainder of this section introduces some notation. Section II introduces the structure-preserving power network model, the Kuramoto model, and different synchronization notions. Section III links the power network model and two variations of the Kuramoto model via topological equivalence. The resulting synchronization conditions for the power network model are stated in Section IV, and Section V concludes the paper.

Geometry on $n$-torus: The torus is the set $\mathbb{T}=]-\pi,+\pi]$, where $-\pi$ and $+\pi$ are associated with each other, an angle is a point $\theta \in \mathbb{T}$, and an arc is a connected subset of $\mathbb{T}$. The product set $\mathbb{T}^{n}$ is the $n$-dimensional torus. With slight abuse of notation, let $\left|\theta_{1}-\theta_{2}\right|$ denote the geodesic distance between two angles $\theta_{1} \in \mathbb{T}$ and $\theta_{2} \in \mathbb{T}$. For $\gamma \in[0, \pi]$, let $\Delta(\gamma) \subset \mathbb{T}^{n}$ be the set of angle arrays $\left(\theta_{1}, \ldots, \theta_{n}\right)$ with the property that there exists an arc of length $\gamma$ containing all $\theta_{1}, \ldots, \theta_{n}$ in its interior. Thus, an angle array $\theta \in \Delta(\gamma)$ satisfies $\max _{i, j \in\{1, \ldots, n\}}\left|\theta_{i}-\theta_{j}\right|<\gamma$. For $\gamma \in[0, \pi]$, we also define $\bar{\Delta}(\gamma)$ to be the union of the phase-synchronized set $\left\{\theta \in \mathbb{T}^{n} \mid \theta_{i}=\theta_{j}, i, j \in\{1, \ldots, n\}\right\}$ and the closure of the open set $\Delta(\gamma)$. Hence, $\theta \in \bar{\Delta}(\gamma)$ satisfies $\max _{i, j \in\{1, \ldots, n\}}\left|\theta_{i}-\theta_{j}\right| \leq \gamma$; the case $\theta \in \bar{\Delta}(0)$ corresponds simply to $\theta$ taking value in the phase-synchronized set.

Vectors and matrices: Given an $n$-tuple $\left(x_{1}, \ldots, x_{n}\right)$, let $x \in \mathbb{R}^{n}$ be the associated vector, and let $\operatorname{diag}\left(\left\{x_{i}\right\}_{i=1}^{n}\right) \in \mathbb{R}^{n}$ be the associated diagonal matrix. The inertia of a matrix $A \in \mathbb{R}^{n \times n}$ are given by the triple $\left\{\nu_{\mathrm{s}}, \nu_{\mathrm{c}}, \nu_{\mathrm{u}}\right\}$, where $\nu_{\mathrm{s}}$ (resp. $\nu_{\mathrm{u}}$ ) denotes the number of stable (resp. unstable) eigenvalues of $A$ in the open left (resp. right) half plane, and $\nu_{\mathrm{c}}$ denotes the number of center eigenvalues with zero real part. The notation blkdiag $\left(A_{1}, \ldots, A_{n}\right)$ denotes the block-diagonal matrix with matrix blocks $A_{1}, \ldots, A_{n}$. Finally, let $\mathbf{1}$ and $\mathbf{0}$ denote the matrices of unit and zero entries of appropriate dimension, and let $I_{n}$ be the $n$-dimensional identity matrix.

Derivative operators: For a twice continuously differentiable function $f: \mathbb{R}^{n} \rightarrow \mathbb{R}$, we adopt the shorthand $\nabla_{i} f(x)=\partial f(x) / \partial x_{i}, \nabla f(x)=(\partial f(x) / \partial x)^{T} \in \mathbb{R}^{n \times 1}$ is the gradient, and $\nabla^{2} f(x) \in \mathbb{R}^{n \times n}$ is the Hessian matrix.

\section{Mathematical Models AND SynCHRonization}

\section{A. Structure-Preserving Power Network Model}

In the following we briefly present the structure-preserving power network model [5] and refer to [12, Chapter 7] for detailed derivation from a higher order first principle model. Let $n>0$ the number of nodes in the power network, among which are $m \in] 0, n$ [ generators and $n-m$ load buses. The network is represented by the symmetric nodal admittance matrix $Y \in \mathbb{C}^{n \times n}$ (augmented with the generator transient reactances), where the indices $\{1, \ldots, m\}$ and $\{m+1, \ldots n\}$ correspond to the generators and loads, respectively. Define the maximum real power transfer between any two nodes $i$ and $j$ with constant positive voltage levels $V_{i}$ and $V_{j}$ as $a_{i j}=V_{i} V_{j} \Im\left(Y_{i j}\right)$ which is positive if $i$ and $j$ are connected and zero otherwise. With this notation the constant-voltage behind reactance swing dynamics of each generator $i$ are

$$
M_{i} \ddot{\theta}_{i}+D_{i} \dot{\theta}_{i}=P_{i}-\sum_{j=1}^{n} a_{i j} \sin \left(\theta_{i}-\theta_{j}\right), i \in\{1, \ldots, m\},
$$

where $\theta_{i} \in \mathbb{T}$ and $\dot{\theta}_{i} \in \mathbb{R}$ are the generator rotor angle and frequency (formulated w.r.t. the net frequency), and $P_{i}>0$, $M_{i}>0$, and $D_{i}>0$ are the mechanical power input, inertia constant, and damping coefficient of generator $i$.

The real power drawn by a load $i \in\{m+1, \ldots, n\}$ consists of a constant term $P_{i}<0$ and a frequency dependent term $D_{i} \dot{\theta}_{i}$, where $D_{i}>0$ and $\theta_{i} \in \mathbb{T}$ is the voltage angle at bus $i$. The resulting in the real power balance equation is

$$
D_{i} \dot{\theta}_{i}=P_{i}-\sum_{j=1}^{n} a_{i j} \sin \left(\theta_{i}-\theta_{j}\right), \quad i \in\{m+1, \ldots, n\} .
$$

The dynamics (1)-(2) evolve in $\mathbb{T}^{n} \times \mathbb{R}^{m}$ and feature an important symmetry, namely the translational invariance of the angular variable $\theta$ on the unit circle $\mathbb{S}^{1}$. This translational invariance can be removed by restricting the dynamics (1)(2) to the quotient space $\mathbb{T}^{n} \backslash \mathbb{S}^{1} \times \mathbb{R}^{m}$. The power network model (1)-(2) can also be formulated with gradient-like and dissipative Hamiltonian (or Newtonian) dynamics as

$$
\begin{aligned}
& M \ddot{\theta}_{i}+D_{i} \dot{\theta}_{i}=P_{i}-\nabla_{i} U(\theta), i \in\{1, \ldots, m\}, \\
& D_{i} \dot{\theta}_{i}=P_{i}-\nabla_{i} U(\theta), \quad i \in\{m+1, \ldots, n\},
\end{aligned}
$$

where $U: \mathbb{T}^{n} \rightarrow \mathbb{R}_{\geq 0}$ is the potential energy of the power flows given by $U(\theta)=1-\sum_{i=1}^{n} \sum_{j=1}^{n} a_{i j} \cos \left(\theta_{i}-\theta_{j}\right)$. The 
representation (3)-(4) reveals that the load frequency coefficients $D_{i}>0$ essentially model dissipation. In the power systems literature, the power inputs $P_{i}$ are traditionally associated with the artificial potential $W:]-\pi, \pi]^{n} \rightarrow \mathbb{R}_{\geq 0}$ defined by $W(\theta)=-\sum_{i=1}^{n} P_{i} \theta_{i}$. Since $W$ is multi-valued on $\mathbb{T}^{n}$, it can be defined only locally on subsets of $\mathbb{T}^{n}$.

In the following we assume that (i) the system parameters are such that the graph induced by the matrix with elements $a_{i j}=a_{j i}$ is connected and (ii) the power network dynamics (1)-(2) feature a finite number of equilibria in the quotient space $\mathbb{T}^{n} \backslash \mathbb{S}^{1} \times \mathbb{R}^{m}$. Of course, both assumption are satisfied for physical power systems, and the second assumption is found to be met for generic parameters $a_{i j}$ [13].

\section{B. Synchronization Notions}

In power systems, synchronization or transient stability are usually defined as stability of an equilibrium of the dynamics (1)-(2) formulated in relative angle coordinates, which include the center of inertia and reference generator coordinates, as well as the infinite bus assumption [6], [12].

Alternatively, the controls, dynamical systems, and the physics community developed and studied various notions of synchronization and incremental stability without employing relative coordinates. The latter concepts are also amenable to relate synchronization to the underlying network topology and parameters. For the power network model (1)-(2) the following synchronization concepts are meaningful, where a synchronized solution has zero angular acceleration (asymptotic linear phase) and an exponential convergence rate due to the locally quadratic nature of the potential function $U(\theta)$.

Definition II.1 (Synchronization) A solution $(\theta, \dot{\theta})$ : $\mathbb{R}_{\geq 0} \rightarrow\left(\mathbb{T}^{n}, \mathbb{R}^{n}\right)$ to the power system model (1)-(2) achieves

1) phase synchronization if there exist constants $\theta_{\text {sync }} \in$ $\mathbb{T}$ and $\dot{\theta}_{\text {sync }} \in \mathbb{R}$ such that all phases $\theta_{i}(t)$ converge exponentially fast to a common linear phase $\theta_{\text {sync }}+$ $\dot{\theta}_{\text {sync }} t(\bmod 2 \pi)$ as $t \rightarrow \infty$;

2) phase-cohesiveness if there exists a length $\gamma \in[0, \pi[$ such that $\theta(t) \in \bar{\Delta}(\gamma)$ for all $t \geq 0$;

3 ) frequency synchronization if there exists a constant $\dot{\theta}_{\text {sync }} \in \mathbb{R}$ such that all frequencies $\dot{\theta}_{i}(t)$ converge exponentially fast to a common constant frequency $\dot{\theta}_{\text {sync }} \in \mathbb{R}$ as $t \rightarrow \infty$; and

4) practical synchronization if it is phase cohesive and it achieves frequency synchronization.

Note that phase synchronization requires exponential stability in the incremental variables $\left|\theta_{i}(t)-\theta_{j}(t)\right|$ and an asymptotically linear phase. We will show that phase synchronization can occur for the power network model (1)-(2) iff all ratios $P_{i} / D_{i}$ are identical. Otherwise, each pairwise distance $\left|\theta_{i}(t)-\theta_{j}(t)\right|$ can converge to a constant value which is not necessarily zero. The concept of phase cohesiveness addresses exactly this point and means that at each time $t$ there exists an arc of length $\gamma$ containing all angles $\theta_{i}(t)$.

Analogously to phase synchronization, the concept of frequency synchronization combines incremental exponential stability together with an asymptotic property. Note that exponential convergence of each frequency $\dot{\theta}_{i}(t)$ to a constant $\dot{\theta}_{\text {sync }} \in \mathbb{R}$ implies that each phase $\theta_{i}(t)=\theta_{i}(0)+\int_{0}^{t} \dot{\theta}_{i}(\tau) d \tau$ converges exponentially to a linear phase $\theta_{i \text {,sync }}+\dot{\theta}_{\text {sync }} t$ $(\bmod 2 \pi)$, where $\theta_{i \text {,sync }} \in \mathbb{T}$ is not necessarily identical for every $i \in\{1, \ldots, n\}$. Finally, practical synchronization combines phase cohesiveness with frequency synchronization. Of course, practical synchronization includes phase synchronization, and, in control-theoretic terms, it corresponds to frequency synchronization with incrementally bounded angles.

\section{Kuramoto Model of Coupled Oscillators}

A celebrated model for the synchronization of coupled oscillators is due to Yoshiki Kuramoto [7]. The Kuramoto model considers $n \geq 2$ coupled oscillators, each represented by a phase variable $\theta_{i} \in \mathbb{T}$ and a natural frequency $\omega_{i} \in \mathbb{R}$. The system of coupled oscillators obeys the dynamics

$$
\dot{\theta}_{i}=\omega_{i}-\frac{K}{n} \sum_{j=1}^{n} \sin \left(\theta_{i}-\theta_{j}\right), \quad i \in\{1, \ldots, n\},
$$

where $K>0$ is the coupling strength. For the Kuramoto model we adopt the synchronization notions in Definition II.1. For identical natural frequencies $\omega_{i}$, the Kuramoto model achieves phase synchronization from almost all initial conditions [14, Theorem 1]. For non-identical natural frequencies, the Kuramoto model can achive only frequency synchronization with a certain level of phase cohesiveness. In particular, the Kuramoto model achieves practical synchronization if and only if the coupling overcomes the worst non-uniformity among the natural frequencies, that is, $K>$ $\max _{i, j \in\{1, \ldots, n\}}\left\{\omega_{i}-\omega_{j}\right\}$, see [10, Theorem 4.1] for details.

Instead of the complete and uniform coupling $K / n$, the Kuramoto model (5) is also studied with general coupling topologies with weights $a_{i j}$. In this case, the dynamics are simply, $\dot{\theta}_{i}=\omega_{i}-\nabla_{i} U(\theta)$ similar to the power network model (3)-(4). The potential function $U(\theta)$ depends on the coupling graph with weights $a_{i j}$, and for a connected and undirected graph the phase-synchronized state is a local minimum of $U(\theta)$ [15, Proposition 3.3.2]. Of particular interest are so-called $\mathbb{S}^{1}$-synchronizing graphs for which the phasesynchronized state is the only local minimum of $U(\theta)$, all other critical points are local maxima or saddle points, and all critical points are hyperbolic. The class of $\mathbb{S}^{1}$-synchronizing graphs is characterized in [15], [16] and references therein, and it includes the complete graph and undirected trees. For these graphs almost global phase synchronization can be concluded by investigating the Hessian of the potential $U(\theta)$.

\section{PARAMETERIZED HAMILTONIAN AND}

\section{GRADIENT-LikE DYNAMICS AND THEIR PROPERTIES}

This section will link the structure-preserving power network model (1)-(2) and the Kuramoto model (5) through a parametrized system. Consider for $n_{1}, n_{2} \geq 0$ and $\lambda \in[0,1]$ the one parameter family $\mathcal{H}_{\lambda}$ of dynamical systems combining dissipative Hamiltonian and gradient-like dynamics as

$$
\begin{aligned}
D_{1} \dot{x}_{1} & =-\nabla_{1} H(x), \\
{\left[\begin{array}{cc}
I_{n_{2}} & \mathbf{0} \\
\mathbf{0} & M
\end{array}\right]\left[\begin{array}{c}
\dot{x}_{2} \\
\dot{x}_{3}
\end{array}\right]=} & \left((1-\lambda)\left[\begin{array}{cc}
\mathbf{0} & I_{n_{2}} \\
-I_{n_{2}} & \mathbf{0}
\end{array}\right]\right. \\
& \left.-\left[\begin{array}{cc}
\lambda D_{2}^{-1} & \mathbf{0} \\
\mathbf{0} & D_{2}
\end{array}\right]\right)\left[\begin{array}{l}
\nabla_{2} H(x) \\
\nabla_{3} H(x)
\end{array}\right],
\end{aligned}
$$


where $x=\left(x_{1}, x_{2}, x_{3}\right) \in \mathcal{X}_{1} \times \mathcal{X}_{2} \times \mathcal{X}_{3}=\mathcal{X}$ is the state, $\mathcal{X}_{1}$ and $\mathcal{X}_{2}$ are smooth manifolds of dimensions ${ }^{1} n_{1}$ and $n_{2}$, the matrices $D_{1} \in \mathbb{R}^{n_{1} \times n_{1}}, D_{2} \in \mathbb{R}^{n_{2} \times n_{2}}$, and $M \in \mathbb{R}^{n_{2} \times n_{2}}$ are positive definite, and $H: \mathcal{X} \rightarrow \mathbb{R}$ is a smooth potential.

The parameterized system (6) continuously interpolates between gradient-like and mixed Hamiltonian/gradient-like dynamics. For $\lambda=1$ system (6) reduces to gradient-like dynamics with a time constant (or system metric) $\mathcal{D}$ as

$$
\mathcal{D} \dot{x}=-\nabla H(x),
$$

where $\mathcal{D} \triangleq \operatorname{blkdiag}\left(D_{1}, D_{2}, D_{2}^{-1} M\right)$. For $\lambda=0$ the dynamics (6) reduce to gradient-like dynamics for $x_{1}$ and dissipative Hamiltonian (or Newtonian) dynamics for $\left(x_{2}, x_{3}\right)$ :

$$
\begin{aligned}
D_{1} \dot{x}_{1} & =-\nabla_{1} H(x) \\
{\left[\begin{array}{cc}
I_{n_{2}} & \mathbf{0} \\
\mathbf{0} & M
\end{array}\right]\left[\begin{array}{l}
\dot{x}_{2} \\
\dot{x}_{3}
\end{array}\right] } & =\left(\left[\begin{array}{cc}
\mathbf{0} & I_{n_{2}} \\
-I_{n_{2}} & \mathbf{0}
\end{array}\right]-\left[\begin{array}{cc}
\mathbf{0} & \mathbf{0} \\
\mathbf{0} & D_{2}
\end{array}\right]\right)\left[\begin{array}{l}
\nabla_{2} H(x) \\
\nabla_{3} H(x)
\end{array}\right] .
\end{aligned}
$$

It turns out that all parameterized systems of the form (6) have the same equilibria, the same local stability properties, and the same global convergence behavior. In particular, the dynamics of (6) are fully governed by potential function $H(x)$. The following theorem summarizes these facts.

Theorem III.1 (Properties of $\mathcal{H}_{\lambda}$ family) Consider for $\lambda \in[0,1]$ the one parameter family $\mathcal{H}_{\lambda}$ of dynamical systems (6). The following statements hold independent of the parameter $\lambda \in[0,1]$ and independent of the particular positive definite matrices $D_{1}, D_{2}, M$ :

1) Equilibria: For all $\lambda \in[0,1]$ the equilibria of $\mathcal{H}_{\lambda}$ are given by the critical points of the potential function $H(x)$, i.e., the set $\mathcal{E} \triangleq\{x \in \mathcal{X}: \nabla H(x)=0\}$.

2) Local stability: For any equilibrium $x^{*} \in \mathcal{E}$ and for all $\lambda \in[0,1]$, the inertia of the Jacobian of $\mathcal{H}_{\lambda}$ are given by the inertia of $-\nabla^{2} H\left(x^{*}\right)$ and the corresponding center-eigenspace is given by $\operatorname{ker} \nabla^{2} H\left(x^{*}\right)$.

3) Global convergence: For any forward-complete solution $x: \mathbb{R}_{>0} \rightarrow \mathcal{X}$ of $\mathcal{H}_{\lambda}$ the function $\tilde{H}(x) \triangleq$ $H\left(x_{1}(t), x_{2}(t), M^{1 / 2} x_{3}(t)\right)$ is non-increasing for all $\lambda \in[0,1]$. In particular, if the sublevel set $\Omega_{c}=$ $\{x \in \mathcal{X}: \tilde{H}(x) \leq c\}$ is compact, then for every initial condition $x(0) \in \Omega_{c}$ the corresponding solution $x: \mathbb{R}_{\geq 0} \rightarrow \Omega_{c}$ is forward-complete and converges to the set $\mathcal{E} \cap \Omega_{c}$, independently of $\lambda \in[0,1]$.

Statements 1) and 2) assert that normal hyperbolicity of the critical points of $H(x)$ can be directly related to local exponential (set) stability for any $\lambda \in[0,1]$. This implies that all vector fields $\mathcal{D}_{\lambda}, \lambda \in[0,1]$, are locally topologically conjugate [17] near a hyperbolic equilibrium point $x^{*} \in \mathcal{E}$. In particular, near $x^{*} \in \mathcal{E}$, trajectories of the gradient vector field (7) can be continuously deformed to match trajectories of the Hamiltonian vector field (8) while preserving parameterization of time. This topological conjugacy holds also for hyperbolic equilibrium trajectories [18, Theorem 6] considered in synchronization. Finally, if the evolution $x(t)$ of the dynamics (6) is bounded, then statement 3) yields global convergence to the equilibria and also suggests $\left(x_{1}, x_{2}, M^{1 / 2} x_{3}\right)$ as natural state space.

\footnotetext{
${ }^{1}$ Note that we also allow $n_{1}=0$ thereby neglecting the $x_{1}$-dynamics.
}

The similarity between second-order Hamiltonian systems and the corresponding first-order gradient flows is wellknown in mechanical control systems [19], in dynamic optimization [20], [21], [22], and also in transient stability studies [23], [24], [25], but we are not aware of any result as general as Theorem III.1. In [23], [24], [25], statements 1) and 2) are proved under the more stringent assumptions that $\mathcal{H}_{\lambda}$ has a finite number of isolated and hyperbolic equilibria. We remark that these assumptions are not met for the power network model (1)-(2) unless the existence of an infinite bus is postulated (a stationary reference node). Additionally, if $H(x)$ constitutes an energy function and if a one-parameter transversality condition is satisfied, then the separatrices of system (6) can also be characterized accurately [23], [24], [26]. Also statement 3) can be refined under further structural assumptions on the potential function $H(x)$, and various other minimizing properties can be deduced from the dynamics (6), see [19], [20], [21], [22].

The proof of Theorem III.1 is presented in the appendix.

\section{A. Equivalence of Local Synchronization}

As a consequence of Theorem III.1, we can link synchronization in the power network model (1)-(2) and in a variant of the Kuramoto model (5). Since Theorem III.1 is valid only for equilibria, we convert synchronization to stability of an equilibrium manifold by transforming to a rotating frame. The explicit synchronization frequency of the power network (1)-(2) is obtained by summing over all equations (1)-(2) as

$$
\sum_{i=1}^{m} M_{i} \ddot{\theta}_{i}+\sum_{i=1}^{n} D_{i} \dot{\theta}_{i}=\sum_{i=1}^{n} P_{i} .
$$

In the frequency-synchronized case, equation (9) simplifies to $\sum_{i=1}^{n} D_{i} \dot{\theta}_{\text {sync }}=\sum_{i=1}^{n} P_{i}$. We conclude that the synchronization frequency is given by $\dot{\theta}_{\text {sync }} \triangleq \sum_{i=1}^{n} P_{i} / \sum_{i=1}^{n} D_{i}$.

Accordingly, define the non-uniform Kuramoto model as

$$
D_{i} \dot{\theta}_{i}=P_{i}-\sum_{j=1}^{n} a_{i j} \sin \left(\theta_{i}-\theta_{j}\right), \quad i \in\{1, \ldots, n\},
$$

and the globally exponentially stable frequency dynamics as

$$
\frac{d}{d t} \dot{\theta}_{i}=-M_{i}^{1} D_{i}\left(\dot{\theta}_{i}-\dot{\theta}_{\mathrm{sync}}\right), \quad i \in\{1, \ldots, m\},
$$

where $D_{i}, P_{i}$, and $a_{i j}$ take the same values as the corresponding parameters for the power network model (1)-(2). It can be easily verified that the non-uniform Kuramoto model (10) has the same synchronization frequency $\dot{\theta}_{\text {sync }}$ as (1)-(2).

Finally, we define the topological Kuramoto model by

$$
\dot{\theta}_{i}=\tilde{P}_{i}-\sum_{j=1}^{n} a_{i j} \sin \left(\theta_{i}-\theta_{j}\right), \quad i \in\{1, \ldots, n\},
$$

where $\tilde{P}_{i} \triangleq P_{i}-D_{i} \dot{\theta}_{\text {sync }}$. Note that the topological Kuramoto model (12) has the same right-hand side as the uniform Kuramoto model (10) formulated in a rotating frame with frequency $\dot{\theta}_{\text {sync }}$ but no multiple rates $D_{i}$ on the left-hand side.

In a rotating frame with frequency $\dot{\theta}_{\text {sync }}$, both the power network model (1)-(2) and the non-uniform Kuramoto model (10) together with frequency dynamics (11) are locally instances of the parameterized system (6) with the potential $H(\theta, \dot{\theta})=T(\dot{\theta})+U(\theta)+W(\theta)$, where $T(\dot{\theta})=\frac{1}{2} \dot{\theta}^{T} \dot{\theta}$ is the normalized kinetic energy of the generator rotors. In the sequel, we seek to apply Theorem III.1 to these two models. 
For a rigorous argumentation, we define a two-parameter family of functions $\phi_{r, s}:\left[0, \infty\left[\rightarrow \mathbb{T}\right.\right.$ of the form $\phi_{r, s}(t) \triangleq$ $r+s \cdot t(\bmod 2 \pi)$, where $r \in \mathbb{T}$ and $s \in \mathbb{R}$. Consider for $\left(r_{1}, \ldots, r_{n}\right) \in \bar{\Delta}(\gamma), \gamma \in[0, \pi[$ the composite function

$$
\Phi_{\gamma, s}:\left[0, \infty\left[\rightarrow \mathbb{T}^{n}, \quad \Phi_{\gamma, s}(t) \triangleq\left(\phi_{r_{1}, s}(t), \ldots, \phi_{r_{n}, s}(t)\right)\right.\right.
$$

mimicking practically-synchronized trajectories of the power network or the Kuramoto models. We now have all ingredients to state the following result on synchronization.

Theorem III.2 (Synchronization Equivalences) Consider the power network model (1)-(2), the non-uniform Kuramoto model (10), and the topological Kuramoto model (12). The following three statements are equivalent for any $\gamma \in[0, \pi[$, $t \in\left[0, \infty\left[\right.\right.$, any function $\Phi_{\gamma, \dot{\theta}_{\mathrm{sync}}}(t)$ as defined in (13):

(i) $\left(\Phi_{\gamma, \dot{\theta}_{\mathrm{sync}}}(t), \dot{\theta}_{\mathrm{sync}} \mathbf{1}_{m}\right)$ parametrizes a locally exponentially stable practically-synchronized trajectory $(\theta(t), \dot{\theta}(t))$ of the power network model (1)-(2).

(ii) $\Phi_{\gamma, \dot{\theta}_{\text {sync }}}(t)$ parametrizes a locally exponentially stable practically-synchronized trajectory $\theta(t)$ of the nonuniform Kuramoto model (10).

(iii) $\Phi_{\gamma, 0}(t)$ parametrizes a locally exponentially stable equilibrium of the topological Kuramoto model (12).

If the equivalent statements (i)-(iii) are true, then, locally near the synchronization manifold $\left(\Phi_{\gamma, \dot{\theta}_{\mathrm{syn}}}(t), \dot{\theta}_{\mathrm{sync}} \mathbf{1}_{m}\right)$, the trajectories of the power network model (1)-(2) are topologically conjugate to the trajectories of the non-uniform Kuramoto model (10) and the frequency dynamics (11).

Proof: By Definition II.1, a practically-synchronized trajectory of the power network model (1)-(2) is exactly characterized by $(\theta(t), \dot{\theta}(t)) \in\left(\Phi_{\gamma, \dot{\theta}_{\text {sync }}}(t), \dot{\theta}_{\text {sync }} \mathbf{1}_{m}\right)$ for $\gamma \in$ $\left[0, \pi\left[\right.\right.$ and $t \geq 0$. In a rotating frame with frequency $\dot{\theta}_{\text {sync }}$, the power network model (1)-(2) takes the form

$$
\begin{aligned}
M \ddot{\theta}_{i}+D_{i} \dot{\theta}_{i}=\tilde{P}_{i}-\nabla_{i} U(\theta), & i \in\{1, \ldots, m\}, \\
D_{i} \dot{\theta}_{i}=\tilde{P}_{i}-\nabla_{i} U(\theta), & i \in\{m+1, \ldots, n\},
\end{aligned}
$$

where $\tilde{P}_{i}=P_{i}-D_{i} \dot{\theta}_{\text {sync }}$. Hence, a practically-synchronized trajectory of (14)-(15) is an equilibrium solution determined up to a translational invariance in $\mathbb{S}^{1}$ and satisfies $(\theta(t), \dot{\theta}(t)) \in\left(\Phi_{\gamma, 0}(t), \mathbf{0}_{m}\right)$. Hence, the phase-synchronized orbit $\left(\Phi_{\gamma, 0}(t), \mathbf{0}_{m}\right)$, understood as a geometric object, constitutes a one-dimensional equilibrium manifold of the power network model (14)-(15) in $\mathbb{T}^{n} \times \mathbb{R}^{n}$. After factoring out the translational invariance, the practically-synchronized orbit $\left(\Phi_{\gamma, 0}(t), \mathbf{0}_{m}\right)$ corresponds to an isolated equilibrium of (14)(15) in the quotient space $\mathbb{T}^{n} \backslash \mathbb{S}^{1} \times \mathbb{R}^{n}$. Since an isolated equilibrium of a smooth nonlinear system with bounded and Lipschitz Jacobian is exponentially stable if and only if the Jacobian is a Hurwitz matrix [27, Theorem 4.15], the locally exponentially stable orbit $\left(\Phi_{\gamma, 0}(t), \mathbf{0}_{m}\right)$ must be hyperbolic in the quotient space. Therefore, the equilibrium trajectory $\left(\Phi_{\gamma, 0}(t), \mathbf{0}_{m}\right)$ is exponentially stable in $\mathbb{T}^{n} \times \mathbb{R}^{m}$ if and only if the Jacobian of (14)-(15) evaluated along $\left(\Phi_{\gamma, 0}(t), \mathbf{0}_{m}\right)$, has $n-1$ stable eigenvalues and one zero eigenvalue corresponding to the translational invariance in $\mathbb{S}^{1}$.
By an analogous argumentation we reach the same conclusion for the non-uniform Kuramoto model (10) written in a rotating frame as $D_{i} \dot{\theta}_{i}=\tilde{P}_{i}-\nabla_{i} U(\theta)$ : the practiallysynchronized trajectory $\Phi_{\gamma, 0}(t) \in \mathbb{T}^{n}$ is exponentially stable if and only if the Jacobian of (10) evaluated along $\Phi_{\gamma, 0}(t)$ has $n-1$ stable eigenvalues and one zero eigenvalue.

Finally, note that both the power network model (14)(15) or the non-uniform Kuramoto model (10), the latter considered in a rotating frame and together with the globally exponentially stable frequency dynamics (11), are locally (near every equilibrium in $\Delta(\gamma) \times \mathbb{R}^{n}$ ) instances of the parameterized system (6) with the potential function $H=T+$ $U+W$. Therefore, by Theorem III.1, the corresponding Jacobians have the same inertia and local exponential stability of one system implies local exponential stability of the other system. This concludes the proof of the equivalence (i) $\Leftrightarrow$ (ii).

The equivalence (ii) $\Leftrightarrow$ (iii) follows simply because the topological Kuramoto model (12) and the non-uniform Kuramoto model (written in a rotating frame) have the same equilibria and their Jacobians $D^{-1} \nabla^{2} U\left(x^{*}\right)$ and $\nabla^{2} U\left(x^{*}\right)$ have the same inertia by Sylvester's inertia theorem [28].

We now prove the final conjugacy statement. By the generalized Hartman-Grobman theorem [18, Theorem 6], the trajectories of (1)-(2) and (10)-(11) (both considered in the rotating frame) are both locally topologically conjugate to the flow generated by their respective linearized vector fields (locally near $\left.\left(\Phi_{\gamma, 0}(t), \mathbf{0}_{m}\right)\right)$. Since both vector fields (1)-(2) and (10)-(11) are hyperbolic with respect to $\left(\Phi_{\gamma, 0}(t), \mathbf{0}_{m}\right)$ and their respective Jacobians have the same hyperbolic inertia (besides the common one-dimensional center eigenspace corresponding to $\left.\left(\Phi_{\gamma, 0}(t), \mathbf{0}_{m}\right)\right)$, both linearized dynamics are topologically conjugate [17, Theorem 7.1]. In summary, we conclude that the trajectories of (1)-(2) and (10)-(11) (in a rotating frame) are locally topologically conjugate near the equilibrium $\left(\Phi_{\gamma, 0}(t), \mathbf{0}_{m}\right)$. In the original non-rotating coordinates, the trajectories of (1)-(2) and (10)-(11) are locally topologically conjugate near $\left(\Phi_{\gamma, \dot{\theta}_{\text {sync }}}(t), \dot{\theta}_{\text {sync }} \mathbf{1}_{m}\right)$.

Remark III.3 (Singular Perturbation Analysis) In [3] we carried out a singular perturbation analysis to relate the power network dynamics (1)-(2) (in network-reduced form) and the non-uniform Kuramoto model (10). For a sufficiently small inertia over damping ratio $\epsilon \triangleq$ $\max _{i \in\{1, \ldots, m\}}\left\{M_{i} / D_{i}\right\}$, the reduced slow dynamics are the non-uniform Kuramoto dynamics (10) and the fast dynamics are the frequency dynamics (11) in the time-scale $t / \epsilon$. By Theorem III.2, the slow and fast dynamics correspond to two decoupled modes, which are topologically conjugate to the power network model. Hence, local equivalence of trajectories of (1)-(2) and (10)-(11) is given by topological conjugacy (without any parametric assumptions), and for $\epsilon$ sufficiently small a dynamic equivalence can be established via singular perturbation analysis as in [3, Theorem IV.2]. $\square$

\section{Fundamental Properties of SynCHRONization}

\section{A. Fundamental Properties of Phase Synchronization}

Even though phase synchronization is not relevant for practical power network applications (due to the resulting 
zero real power flows), its analysis yields important insights. For zero power inputs $P_{i}=0$, the Hamiltonian formulation (3)-(4) of the power network model and Theorem III.1 imply that phase synchronization depends exclusively on the Hamiltonian $T(\dot{\theta})+U(\theta)$ : local maxima and saddle points obviously correspond to unstable states and local minima to stable states. The following result confirms this intuition and also shows the crucial role of the dissipation terms $D_{i}$.

Theorem IV.1 (Exponential phase synchronization) Consider the power network model (1)-(2).

The following two statements are equivalent

(i) Local phase synchronization: the phase-synchronized state is locally exponentially and has a constant synchronization frequency $\dot{\theta}_{\text {sync }} \in \mathbb{R}$;

(ii) Uniformity: there exists a constant $\bar{P} \in \mathbb{R}$ such that $P_{i}=D_{i} \bar{P}$ for all $i \in\{1, \ldots, n\}$.

Moreover, in either of the two equivalent cases (i) or (ii), $\bar{P} \equiv \dot{\theta}_{\text {sync }}$ and the following three statements hold:

1) Explicit phase: The asymptotic synchronization phase is given by $\sum_{i=1}^{n} D_{i} \theta_{i}(0) / \sum_{i=1}^{n} D_{i}+\dot{\theta}_{\text {sync }} t(\bmod 2 \pi)$.

2) Global convergence: For all initial conditions $(\theta(0), \dot{\theta}(0)) \in \mathbb{T}^{n} \times \mathbb{R}^{m}$ all frequencies $\dot{\theta}_{i}(t)$ converge to $\bar{P}$ and all phases $\theta_{i}(t)-\dot{\theta}_{\text {sync }} t(\bmod 2 \pi)$ converge to the critical points of the potential function $U(\theta)$.

3) Almost global stability: If the graph induced by $a_{i j}$ is $\mathbb{S}^{1}$-synchronizing, then for almost all initial conditions $(\theta(0), \dot{\theta}(0)) \in \mathbb{T}^{n} \times \mathbb{R}^{m}$, the phases of the power network model synchronize exponentially.

Proof: We first proof the implication (i) $\Longrightarrow$ (ii). In the phase-synchronized case, there exist constants $\theta_{\text {sync }} \in \mathbb{T}$ and $\dot{\theta}_{\text {sync }} \in \mathbb{R}$ such that $\theta_{i}(t)=\theta_{\text {sync }}+\dot{\theta}_{\text {sync }} t, \dot{\theta}_{i}(t)=\dot{\theta}_{\text {sync }}$, and $\ddot{\theta}_{i}(t)=0$ for all $i \in\{1, \ldots, n\}$. Hence, the dynamics (1)-(2) read as $D_{i} \dot{\theta}_{\text {sync }}=P_{i}$ for all $i \in\{1, \ldots, n\}$. Hence, a necessary condition for the existence of phase-synchronized solutions is that $P_{i} / D_{i}=\dot{\theta}_{\text {sync }}$ is constant for $i \in\{1, \ldots, n\}$.

For the implication (ii) $\Longrightarrow$ (i) we transform the model (1)-(2) to a rotating frame with frequency $\dot{\theta}_{\text {sync }}$ as

$$
\begin{aligned}
& M \ddot{\theta}_{i}+D_{i} \dot{\theta}_{i}=-\nabla_{i} U(\theta), i \in\{1, \ldots, m\}, \\
& D_{i} \dot{\theta}_{i}=-\nabla_{i} U(\theta), \quad i \in\{m+1, \ldots, n\} .
\end{aligned}
$$

By Theorem III.2, we conclude that the phase-synchronized state of (16)-(17) is locally exponentially stable if and only if the the phase-synchronized state of the corresponding topological Kuramoto model (12) (with $\tilde{P}_{i}=0$ by assumption) is exponentially stable. The latter is true for a connected graph [15, Proposition 3.3.2]. This concludes the proof of (ii) $\Leftrightarrow$ (i).

To prove statement 1), we sum over all equations (16)-(17):

$$
\sum_{i=1}^{m} M_{i} \ddot{\theta}_{i}+\sum_{i=1}^{n} D_{i} \dot{\theta}_{i}=0 .
$$

Integration of equation (18) along phase-synchronized solutions yields that $\sum_{i=1}^{n} D_{i} \theta_{i}(t)=\sum_{i=1}^{n} D_{i} \theta_{i}(0)$ is constant for all $t \geq 0$, where we already accounted for $\dot{\theta}_{i}(t)=0$ for all $i \in\{1, \ldots, n\}$ and all $t \geq 0$. Hence, the synchronization phase is given by a weighted average of the initial conditions $\sum_{i=1}^{n} D_{i} \theta_{i}(0) / \sum_{i=1}^{n} D_{i}$. In the original coordinates (nonrotating frame) the synchronization phase is then given by $\sum_{i=1}^{n} D_{i} \theta_{i}(0) / \sum_{i=1}^{n} D_{i}+\dot{\theta}_{\text {sync }} t$, which proves statement 1$)$.

Since the level sets of $T\left(M^{1 / 2} \dot{\theta}\right)+U(\theta)$ are compact, Theorem III.1 can be applied to system (16)-(17) and yields convergence to the critical points of the $T\left(M^{1 / 2} \dot{\theta}\right)+U(\theta)$. Consequently, we obtain statement 2) in the rotating frame.

By statement 2), all solutions of (16)-(17) converge to the set of equilibria. By Theorem III.2, these equilibria are exponentially stable if and only if the corresponding equilibria of the topological Kuramoto model (12) are exponentially stable. Statement 3) follows since all non-phase-synchronized equilibria are unstable for $\mathbb{S}^{1}$-synchronizing graphs

\section{B. Fundamental Properties of Practical Synchronization}

According to Theorem IV.1, phase synchronization occurs if and only if all ratios $P_{i} / D_{i}$ are identical. This intuition carries over to a necessary conditions for frequency synchronization. In particular, frequency synchronization of a node $i$ with the rest of the network cannot occur if the coupling of node $i$ to the network via the power transfers $\sum_{j=1}^{n} a_{i j}$ is too weak and the ratio of power input and dissipation, $P_{i} / D_{i}$, diverges too much from the weighted average $\dot{\theta}_{\text {sync }}$.

Lemma IV.2 (Necessary conditions for frequency synchronization) Consider the power network model (1)-(2). If the power network achieves frequency synchronization, then the following two statements hold:

1) Explicit frequency: the asymptotic synchronization frequency is given by $\dot{\theta}_{\text {sync }} \triangleq \sum_{k=1}^{n} P_{k} / \sum_{k=1}^{n} D_{k}$.

2) Uniformity: for all $i \in\{1, \ldots, n\}$ it holds that

$$
\sum_{j=1}^{n} a_{i j} \geq\left|P_{i}-D_{i} \dot{\theta}_{\text {sync }}\right| \text {. }
$$

Proof: Statement 1) has already been proven in Subsection III-A. To prove statement 2) consider the power network model (1)-(2) and assume the transformation to a rotating frame with frequency $\dot{\theta}_{\text {sync }}=\sum_{i=1}^{n} P_{i} / \sum_{i=1}^{n} D_{i}$ has been carried out. For a frequency-synchronized solutions, the transformed power network dynamics (written in a rotating frame) must be in an equilibrium determined by

$$
0=P_{i}-D_{i} \dot{\theta}_{\mathrm{sync}}-\sum_{j=1}^{n} a_{i j} \sin \left(c_{i j}\right), \quad i \in\{1, \ldots, n\},
$$

where $c_{i j} \in \mathbb{T}$ for $i, j \in\{1, \ldots, n\}$. Note that the previous equation has no solution if condition (19) does not hold.

Before stating conditions for practical synchronization of the power network model (1)-(2), we first consider the simpler case of a uniform power network, where all coupling coefficients take the value $a_{i j}=K / n$ for $K>0$ and $i, j \in\{1, \ldots, n\}$. The uniform power network model reads as

$$
M_{i} \ddot{\theta}_{i}+D_{i} \dot{\theta}_{i}=P_{i}-\frac{K}{n} \sum_{j=1}^{n} \sin \left(\theta_{i}-\theta_{j}\right), i \in\{1, \ldots, m\},
$$

$$
D_{i} \dot{\theta}_{i}=P_{i}-\frac{K}{n} \sum_{j=1}^{n} \sin \left(\theta_{i}-\theta_{j}\right), i \in\{m+1, \ldots, n\} \text {, }
$$


where $M_{i}, D_{i}$, and $P_{i}$ take the same values as the corresponding parameters in the original power network model (1)-(2). For the uniform power network model we can state exact synchronization conditions which generalize the results [10] known for the classic Kuramoto model (5) to multi-rate and second-order Kuramoto models with inertia and damping.

Theorem IV.3 (Practical synchronization in a uniform power network model) Consider the uniform power network model (20)-(21), and define the scaled power inputs $\tilde{P}_{i}=$ $P_{i}-D_{i} \dot{\theta}_{\text {sync }}$, where $\dot{\theta}_{\text {sync }}=\sum_{k=1}^{n} P_{k} / \sum_{k=1}^{n} D_{k}$.

The following two statements are equivalent:

(i) The coupling strength $K$ is larger than the maximum non-uniformity among the scaled power inputs, i.e., $K>K_{\text {critical }}:=\max _{i, j \in\{1, \ldots, n\}}\left\{\tilde{P}_{i}-\tilde{P}_{j}\right\}$.

(ii) There exists a locally exp. stable practically synchronized solution of the uniform power network model.

Moreover, in either of the two equivalent cases (i) and (ii), the practically synchronized solution has the frequency $\dot{\theta}_{\text {sync }}$ and it is phase cohesive in $\bar{\Delta}\left(\gamma_{\min }\right)$, where $\gamma_{\min } \in[0, \pi / 2[$ is the unique solution to $\sin \left(\gamma_{\min }\right)=K_{\text {critical }} / K$.

Proof: By Theorem III.2, a locally exponentially stable practically synchronized trajectory of the uniform power network model (20)-(21) exists if and only if there exists a locally exponentially stable equilibrium of the corresponding topological Kuramoto model (12), with natural frequencies $\tilde{P}_{i}=P_{i}-D_{i} \dot{\theta}_{\text {sync }}$ and uniform coupling $a_{i j}=K / n$ for all $i, j \in\{1, \ldots, n\}$. The latter is true if and only if statement (i) holds [10, Theorem 4.1]. Moreover, [10, Theorem 4.1] asserts that a practically synchronized solution is phase cohesive in $\bar{\Delta}\left(\gamma_{\min }\right)$ with $\gamma_{\min }$ as stated above.

Lemma IV.2 and Theorem IV.3 give the intuition that "the coupling via $a_{i j}$ has to dominate the non-uniformity in $P_{i} / D_{i}$ " for synchronization. This intuition is confirmed by a sufficient synchronization condition for the original power network model (1)-(2), where the coupling strength is quantified in terms of the algebraic connectivity $\lambda_{2}\left(L\left(a_{i j}\right)\right)$, i.e., the second smallest eigenvalue of the Laplacian $L\left(a_{i j}\right)=$ $\operatorname{diag}\left(\left\{A_{i i}\right\}_{i=1}^{n}\right)-A$ of the weighted power network graph.

Theorem IV.4 (Sufficient conditions for frequency synchronization) Consider the power network model (1)(2). Assume that the graph induced by $a_{i j}$ is connected and has algebraic connectivity $\lambda_{2}\left(L\left(a_{i j}\right)\right)$. Define the scaled power inputs $\tilde{P}_{i}=P_{i}-D_{i} \dot{\theta}_{\text {sync }}$, where $\dot{\theta}_{\text {sync }}=$ $\sum_{k=1}^{n} P_{k} / \sum_{k=1}^{n} D_{k}$. Assume that the algebraic connectivity dominates the non-uniformity in scaled power inputs, i.e.,

$$
\lambda_{2}\left(L\left(a_{i j}\right)\right)>\lambda_{\text {critical }}:=\left(\sum_{i, j=1, i<j}^{n}\left|\tilde{P}_{i}-\tilde{P}_{j}\right|^{2}\right)^{1 / 2} .
$$

Then the practically synchronized state is locally exponentially stable with synchronization frequency $\dot{\theta}_{\text {sync }}$ and phase cohesiveness in $\bar{\Delta}\left(\gamma_{\min }\right)$ with $\gamma_{\min } \in[0, \pi / 2[$ given as the unique solution to $\sin \left(\gamma_{\text {min }}\right)=\lambda_{\text {critical }} / \lambda_{2}\left(L\left(a_{i j}\right)\right)$.

Proof: By Theorem III.2, the practically synchronized state of the power network model (1)-(2) is locally exponen- tially stable if and only if the practically-synchronized equilibrium of the topological Kuramoto model (12) is locally exponentially stable with $\tilde{P}_{i}=P_{i}-D_{i} \dot{\theta}_{\text {sync }}$. A sufficient condition for the latter to be true is given by condition (22), as proved in [3, Theorem V.5]. Moreover, [3, Theorem V.5] asserts that a practically synchronized solution is phase cohesive in $\bar{\Delta}\left(\gamma_{\min }\right)$ with $\gamma_{\min }$ as stated above.

\section{CONCLUSiOnS}

In this paper showed the equivalence of exponential synchronization in a structure-preserving power network model, exponential synchronization in a reduced order non-uniform Kuramoto model, and exponential stability of a topological Kuramoto model. This equivalence can be interpreted geometrically as topological conjugacy, and, based on this equivalence, we presented various results for phase, frequency, and practical synchronization in the power network model.

Our results highlight the crucial role of the dissipation terms, power inputs, and the network topology. The insights gained in this paper may be beneficial develop new strategies for coordinated controller design and controlled islanding in a power network. The presented results are only a first step and should be extended towards exact and non-local synchronization conditions for more detailed power system models.

\section{APPENDIX}

Proof of Theorem III.1: To prove statement 1), we reformulate the dynamics (6) as

$$
\left[\begin{array}{c}
\dot{x}_{1} \\
\dot{x}_{2} \\
M \dot{x}_{3}
\end{array}\right]=-\underbrace{\left[\begin{array}{ccc}
D_{1}^{-1} & \mathbf{0} & \mathbf{0} \\
\mathbf{0} & \lambda D_{2}^{-1} & -(1-\lambda) I_{n_{2}} \\
\mathbf{0} & (1-\lambda) I_{n_{2}} & D_{2}
\end{array}\right]}_{\triangleq W_{\lambda}}\left[\begin{array}{c}
\nabla_{1} H(x) \\
\nabla_{2} H(x) \\
\nabla_{3} H(x)
\end{array}\right] .
$$

It follows from the Schur determinant formula [29] that $\operatorname{det}\left(W_{\lambda}\right)=\operatorname{det}\left(D_{1}^{-1}\right)\left(\lambda+(1-\lambda)^{2}\right)$ is positive for all $\lambda \in[0,1]$. Hence, $W_{\lambda}$ is nonsingular for all $\lambda \in[0,1]$, and the equilibria of (6) are given by $\nabla H(x)=\mathbf{0}$.

To prove statement 2) we analyze the Jacobian of $\mathcal{H}_{\lambda}$ at an equilibrium $x^{*} \in \mathcal{E}$ given by

$$
\begin{gathered}
J_{\lambda}\left(x^{*}\right)=\underbrace{\left[\begin{array}{cc|c}
D_{1}^{-1} & 0 & 0 \\
0 & \lambda D_{2}^{-1} & (\lambda-1) M^{-1} \\
\hline 0 & (1-\lambda) M^{-1} & M^{-1} D_{2} M^{-1}
\end{array}\right]}_{\triangleq S_{\lambda}} \\
\underbrace{\left[\begin{array}{c|c}
-I_{n_{1}+n_{2}} & \mathbf{0} \\
\hline \mathbf{0} & -M
\end{array}\right] \nabla^{2} H\left(x^{*}\right)}_{\triangleq S\left(x^{*}\right)} .
\end{gathered}
$$

Similar to the proof of statement 1), we see that $\operatorname{det}\left(S_{\lambda}\right)=$ $\operatorname{det}\left(D_{1}^{-1}\right) \operatorname{det}\left(D_{2}^{-1}\right) \operatorname{det}\left(M^{-1} D_{2} M^{-1}\right)\left(\lambda+(1-\lambda)^{2}\right)$. Thus, $S_{\lambda}$ is nonsingular for $\lambda \in[0,1]$, and the nullspace of $J_{\lambda}\left(x^{*}\right)$ is given by $\operatorname{ker} \nabla^{2} H\left(x^{*}\right)$ (independently of $\lambda \in[0,1]$ ).

To show that the stability properties of the equilibrium $x^{*} \in \mathcal{E}$ are independent of $\lambda \in[0,1]$, we prove that the inertia of the Jacobian $J_{\lambda}\left(x^{*}\right)$ depend only on $S\left(x^{*}\right)$ and not on $\lambda \in[0,1]$. For the invariance of the inertia we appeal to the main inertia theorem for positive semi-definite matrices [28, Theorem 5]. Note that $J_{\lambda}\left(x^{*}\right)$ and $J_{\lambda}\left(x^{*}\right)^{T}$ have the 
same eigenvalues. Let $A \triangleq J_{\lambda}\left(x^{*}\right)^{T}$ and $P \triangleq S\left(x^{*}\right)$, and consider the matrix $Q$ obtained by the Lyapunov equality as

$$
Q=\frac{1}{2}\left(A P+P A^{T}\right)=P\left[\begin{array}{ccc}
D_{1}^{-1} & 0 & 0 \\
0 & \lambda D_{2}^{-1} & 0 \\
0 & 0 & M^{-1} D_{2} M^{-1}
\end{array}\right] P .
$$

Note that $Q$ is positive semidefinite for $\lambda \geq 0$, and for $\lambda \neq 0$ the nullspace of $Q$ coincides with the nullspace of $P$, i.e., $\operatorname{ker} Q=\operatorname{ker} P$. Hence, for $\lambda \in] 0,1]$ the assumptions of $[28$, Theorem 5] are satisfied, and it follows that the non-zero inertia of $A=J_{\lambda}\left(x^{*}\right)^{T}$ (restricted to image of $A$ ) correspond to the non-zero inertia of $P$. Hence, the nonzero inertia of $J_{\lambda}\left(x^{*}\right)$ are independent of $\left.\left.\lambda \in\right] 0,1\right]$, and possible zero eigenvalues correspond to $\operatorname{ker} P=\operatorname{ker} \nabla^{2} H\left(x^{*}\right)$. To handle the case $\lambda=0$ we invoke continuity arguments. Since the eigenvalues of $J_{\lambda}\left(x^{*}\right)$ are continuous functions of the matrix elements, the inertia of $J_{0}\left(x^{*}\right)$ are the same as the inertia of $J_{\lambda}\left(x^{*}\right)$ for $\lambda>0$ sufficiently small.

In summary, the inertia of $J_{\lambda}\left(x^{*}\right)$ are given by the inertia of $P$ independent of $\lambda \in[0,1]$. From Sylvester's inertia theorem [28] and since blkdiag $\left(I_{n_{1}+n_{2}}, M\right)$ is positive definite, the inertia of $P=\operatorname{blkdiag}\left(I_{n_{1}+n_{2}}, M\right)\left(-\nabla^{2} H\left(x^{*}\right)\right)$ are again given by the inertia of $-\nabla^{2} H\left(x^{*}\right)$. In conclusion, the inertia and the nullspace of $J_{\lambda}\left(x^{*}\right)$ are given by the inertia of $-\nabla^{2} H\left(x^{*}\right)$ and $\operatorname{ker} \nabla^{2} H\left(x^{*}\right)$.

To prove statement 3 ) consider the positive definite square root $M^{1 / 2}$ of $M$. Inspired by [23], we apply the similarity transformation $z=T x$ with $T=\operatorname{blkdiag}\left(I_{n_{1}}, I_{n_{2}}, M^{1 / 2}\right)$. In $z$-coordinates the dynamics (6) read as

$$
\left[\begin{array}{c}
\dot{z}_{1} \\
\dot{z}_{2} \\
\dot{z}_{3}
\end{array}\right]=-\left[\begin{array}{ccc}
D_{1}^{-1} & \mathbf{0} & \mathbf{0} \\
\mathbf{0} & \lambda D_{2}^{-1} & -(1-\lambda) M^{-1 / 2} \\
\mathbf{0} & (1-\lambda) M^{-1 / 2} & M^{-1 / 2} D_{2} M^{-1 / 2}
\end{array}\right] \nabla V(z),
$$

where $V(z) \triangleq H\left(T^{-1} z\right)$ is the potential in $z$-coordinates. The derivative of $V(z)$ along trajectories of (24) is given by

$\dot{V}(z)=-\nabla V(z)^{T} \operatorname{blkdiag}\left(D_{1}^{-1}, \lambda D_{2}^{-1}, M^{-1 / 2} D_{2} M^{-1 / 2}\right) \nabla V(z)$.

Thus, $V(z(t))$ is non-increasing along solutions of (24) and its sublevel sets $\tilde{\Omega}_{c}=\{z \in T \mathcal{X}: V(z) \leq c\}_{\sim}$ are positively invariant. Equivalently in $x$-coordinates, $\tilde{H}(x(t))=$ $H\left(x_{1}(t), x_{2}(t), M^{1 / 2} x_{3}(t)\right)$ is non-increasing along solutions of (6) and $\Omega_{c}=T^{-1} \circ \tilde{\Omega}_{c}$ is positively invariant.

If additionally the sublevel sets $\Omega_{c}$ are compact, then every solution $x(t)$ of the smooth dynamics (6) with initial condition $x(0) \in \Omega_{c}$ is forward-complete [27, Theorem 3.3]. The same properties can be asserted in $z$-coordinates. Furthermore, the invariance principle [27, Theorem 4.4], applied in $z$-coordinates, asserts that every solution $z(t)$ with initial condition $z(0)$ in the compact and positively invariant set $\tilde{\Omega}_{c}=T \circ \Omega_{c}$ converges to the largest invariant set contained in $\left\{z \in \tilde{\Omega}_{c}: \nabla_{1} V(z)=\mathbf{0}, \lambda \nabla_{2} V(z)=\mathbf{0}, \nabla_{3} V(z)=\mathbf{0}\right\}$, i.e., the set where $\dot{V}(z)=0$. Hence, for $\lambda \in] 0,1]$ the positive limit sets of (24) are clearly given by $T \circ\left(\Omega_{c} \cap \mathcal{E}\right)$. For $\lambda=0$, the largest set contained in $\dot{V}(z)=0$, which is also invariant w.r.t. the dynamics (24), is obviously again the set $T \circ\left(\Omega_{c} \cap \mathcal{E}\right)$. In original $x$-coordinates we conclude that the positive limit set of the dynamics (6) is the set $\Omega_{c} \cap \mathcal{E}$. This completes the proof of Theorem III.1.

\section{REFERENCES}

[1] I. A. Hiskens, "Analysis tools for power systems-contending with nonlinearities," Proceedings of the IEEE, vol. 83, no. 11, pp. 15731587,2002

[2] D. J. Hill and G. Chen, "Power systems as dynamic networks," in IEEE Int. Symposium on Circuits and Systems, Kos, Greece, May 2006, pp. $722-725$.

[3] F. Dörfler and F. Bullo, "Synchronization and transient stability in power networks and non-uniform Kuramoto oscillators," IEEE Transactions on Automatic Control, Jan. 2010, submitted.

[4] , "Kron reduction of graphs with applications to electrical networks," SIAM Review, Feb. 2011, submitted.

[5] A. R. Bergen and D. J. Hill, "A structure preserving model for power system stability analysis," IEEE Transactions on Power Apparatus and Systems, vol. 100, no. 1, pp. 25-35, 1981 .

[6] H.-D. Chiang, C. C. Chu, and G. Cauley, "Direct stability analysis of electric power systems using energy functions: theory, applications, and perspective," Proceedings of the IEEE, vol. 83, no. 11, pp. 1497$1529,1995$.

[7] Y. Kuramoto, "Self-entrainment of a population of coupled nonlinear oscillators," in Int. Symposium on Mathematical Problems in Theoretical Physics, ser. Lecture Notes in Physics, H. Araki, Ed. Springer, 1975 , vol. 39 , pp. $420-422$.

[8] S. H. Strogatz, "From Kuramoto to Crawford: Exploring the onset of synchronization in populations of coupled oscillators," Physica D: Nonlinear Phenomena, vol. 143, no. 1, pp. 1-20, 2000.

[9] J. A. Acebron, L. L. Bonilla, C. J. P. Vicente, F. Ritort, and R. Spigler, "The Kuramoto model: A simple paradigm for synchronization phenomena," Reviews of Modern Physics, vol. 77, no. 1, pp. 137-185, 2005 .

[10] F. Dörfler and F. Bullo, "On the critical coupling for Kuramoto oscillators," SIAM Journal on Applied Dynamical Systems, Nov. 2010, submitted.

[11] J. Zhu, Y. Tian, and J. Kuang, "On the general consensus protocol of multi-agent systems with double-integrator dynamics," Linear Algebra and its Applications, vol. 431, no. 5-7, pp. 701-715, 2009.

[12] P. W. Sauer and M. A. Pai, Power System Dynamics and Stability. Prentice Hall, 1998.

[13] J. Baillieul and C. Byrnes, "Geometric critical point analysis of lossless power system models," IEEE Transactions on Circuits and Systems, vol. 29, no. 11, pp. 724-737, 1982.

[14] R. Sepulchre, D. A. Paley, and N. E. Leonard, "Stabilization of planar collective motion: All-to-all communication," IEEE Transactions on Automatic Control, vol. 52, no. 5, pp. 811-824, 2007.

[15] A. Sarlette, "Geometry and symmetries in coordination control," Ph.D. dissertation, University of Liège, Belgium, Jan. 2009.

[16] E. Canale, P. Monzon, and F. Robledo," "On the complexity of the classification of synchronizing graphs," in Grid and Distributed Computing, Control and Automation: International Conferences, Jeju Island, Korea, Dec. 2010, p. 186.

[17] C. Robinson, Dynamical systems: stability, symbolic dynamics, and chaos. CRC Press, 1999.

[18] E. A. Coayla-Teran, S. E. A. Mohammed, and P. R. C. Ruffino, "Hartman-Grobman theorems along hyperbolic stationary trajectories," Dynamical Systems, vol. 17, no. 2, pp. 281-292, 2007.

[19] D. E. Koditschek, "The application of total energy as a Lyapunov function for mechanical control systems," in Dynamics and Control of Multibody Systems, J. E. Marsden, P. S. Krishnaprasad, and J. C. Simo, Eds. AMS, 1989, vol. 97, pp. 131-157.

[20] F. Alvarez, "On the minimizing property of a second order dissipative system in Hilbert spaces," SIAM Journal on Control and Optimization, vol. 38 , no. 4 , pp. $1102-1119,2000$

[21] H. Attouch and P. Mainge, "Asymptotic behavior of second-order dissipative evolution equations combining potential with non-potential effects," ESAIM: Control, Optimisation and Calculus of Variations, 2010, to appear.

[22] X. Goudou and J. Munier, "The gradient and heavy ball with friction dynamical systems: the quasiconvex case," Mathematical Programming, vol. 116, no. 1, pp. 173-191, 2009.

[23] H. D. Chiang and F. F. Wu, "Stability of nonlinear systems described by a second-order vector differential equation," IEEE Transactions on Circuits and Systems, vol. 35, no. 6, pp. 703-711, 2002.

[24] H.-D. Chiang, F. F. Wu, and P. P. Varaiya, "A BCU method for direct analysis of power system transient stability," IEEE Transactions on Power Systems, vol. 9, no. 3, pp. 1194-1208, 1994.

[25] C. Chu, "Transient dynamics of electric power systems: Direct stability assessment and chaotic motions," Ph.D. dissertation, Cornell University, 1996.

[26] H.-D. Chiang and C. C. Chu, "Theoretical foundation of the BCU method for direct stability analysis of network-reduction power system models with small transfer conductances," IEEE Transactions on Circuits and Systems I: Fundamental Theory and Applications, vol. 42, no. 5, pp. 252-265, 1995.

[27] H. K. Khalil, Nonlinear Systems, 3rd ed. Prentice Hall, 2002.

[28] D. Carlson and H. Schneider, "Inertia Theorems for Matrices: The Semidefinite Case," Journal of Mathematical Analysis and Applications, vol. 6, pp. 430-446, 1963

[29] F. Zhang, The Schur Complement and its Applications. Springer, 2005. 